PROCEEDINGS OF THE

AMERICAN MATHEMATICAL SOCIETY

Volume 129, Number 9, Pages 2679-2687

S 0002-9939(01)05897-X

Article electronically published on February 9, 2001

\title{
NONCOMMUTATIVE COMPLEX ANALYSIS AND BARGMANN-SEGAL MULTIPLIERS
}

\author{
RICHARD ROCHBERG AND NIK WEAVER
}

(Communicated by David R. Larson)

\begin{abstract}
We state several equivalent noncommutative versions of the Cauchy-Riemann equations and characterize the unbounded operators on $L^{2}(\mathbf{R})$ which satisfy them. These operators arise from the creation operator via a functional calculus involving a class of entire functions, identified by Newman and Shapiro, which act as unbounded multiplication operators on Bargmann-Segal space.
\end{abstract}

\section{Noncommutative Cauchy-Riemann equations}

Various branches of the field of operator algebras are, following Connes [7, Effros [8], Woronowicz [18], and others, often viewed as "noncommutative" or "quantized" versions of classical mathematical subjects. Underlying this attitude is a rather diverse family of fundamental examples, of which the noncommutative tori [16] and the noncommutative plane $([12],[13])$ are probably the most basic. Our aim here is to determine an appropriate version of complex structure on the latter.

The basic elements of the noncommutative plane are these. The underlying set is the Hilbert space $L^{2}(\mathbf{R})$. Compact operators play the role of continuous functions which vanish at infinity and bounded operators play the role of bounded measurable functions. The unbounded self-adjoint operators $Q=M_{x}$ (multiplication by $x$ ) and $P=-i \hbar \frac{d}{d x}$, where $\hbar$ is a nonzero real number, are analogous to the coordinate functions, and commutation with the operators $-\frac{1}{\hbar} P$ and $\frac{1}{\hbar} Q$ correspond to the partial derivatives $\frac{\partial}{\partial x}$ and $\frac{\partial}{\partial y}$.

Physically, this structure characterizes a spinless, nonrelativistic, one-dimensional quantum-mechanical particle. The "noncommutative plane" intuition is motivated by the fact that the phase space of a classical one-dimensional particle is the ordinary plane, the classical position and momentum observables are the coordinate functions on $\mathbf{R}^{2}$, and so on.

In what sense does the noncommutative plane carry a complex structure? Consider a differentiable function $\phi: \mathbf{C} \rightarrow \mathbf{C}$. By regarding it as a function from $\mathbf{R}^{2}$ into $\mathbf{C}$, we may condense the Cauchy-Riemann equations, which diagnose whether $\phi$ is holomorphic, into the single condition

$$
\frac{\partial \phi}{\partial y}=i \frac{\partial \phi}{\partial x}
$$

Received by the editors September 27, 1999 and, in revised form, January 14, 2000.

2000 Mathematics Subject Classification. Primary 46L89, 47B32; Secondary 30D15.

(C)2001 American Mathematical Society 
In terms of the unbounded multiplication operator $M_{\phi}: f \rightarrow \phi f$ acting on $L^{2}\left(\mathbf{R}^{2}\right)$ this condition can formally be reexpressed as

$$
\left[M_{\phi}, \frac{\partial}{\partial x}+i \frac{\partial}{\partial y}\right]=0
$$

or equivalently

$$
\left[M_{\phi}, \frac{\partial}{\partial y}-i \frac{\partial}{\partial x}\right]=0 .
$$

(The general principle is that $\left[M_{\phi}, \frac{\partial}{\partial x}\right]=-M_{\partial \phi / \partial x}$, etc.)

Now the noncommutative analogs of $\frac{\partial}{\partial x}$ and $\frac{\partial}{\partial y}$ acting on functions are commutation with $-\frac{1}{\hbar} P$ and $\frac{1}{\hbar} Q$ acting on operators. So, depending on whether we identify position with the $x$-axis and momentum with the $y$-axis or vice versa, the noncommutative version of the Cauchy-Riemann equations is either the condition

$$
\left[A, a^{-}\right]=0
$$

or the condition

$$
\left[A, a^{+}\right]=0
$$

where $a^{-}$is the annihilation operator $a^{-}=\frac{1}{2}(Q+i P)$ and $a^{+}$is the creation operator $a^{+}=\frac{1}{2}(Q-i P)$. Either condition is to be satisfied by a possibly unbounded operator $A$ acting on $L^{2}(\mathbf{R})$.

In classical complex analysis the variables can be switched without essentially changing the theory, but in the noncommutative setting this is not true: the creation and annihilation operators are not equivalent. However, if one uses the right domains they are each other's adjoints, so the class of operators which satisfy $(*)$ ought to be adjoint to the class of operators which satisfy $(* *)$.

This is not a rigorous assertion since $(*)$ and $(* *)$ are only formal expressions; there is no standard way to define the commutator of two unbounded operators. In fact, dealing with that general issue in this particular context is one of the main technical themes of this paper. Our intention is to do it in a way which allows us to include a large natural class of operators and in a way which insures that the basic facts expected from formal computations will hold. That work is in Section 3. In Section 4 we have a brief discussion of the difficulties which arise when one tries to work with too large a class of operators.

To make these conditions precise we need a clearer picture of the operators $a^{+}$ and $a^{-}$. Mathematically, the nicest representation of the creation and annihilation operators - and the setting in which their natural domains are apparent — is on Bargmann-Segal space, so we go there to pursue the question further.

\section{Bargmann-Segal Formulation}

From now on we set $r=1 / \hbar$ and assume $r>0$. (Changing the sign of $\hbar$ is tantamount to interchanging $P$ and $Q$.) The Bargmann-Segal space, also known as Bargmann-Fock or Fischer space, is the Hilbert space $\mathcal{F}$ consisting of all entire functions on the complex plane which are square-integrable with respect to the Gaussian measure $\mu$ defined by $d \mu(z)=(r / \pi) e^{-r|z|^{2}} d z$. It is isomorphic to $L^{2}(\mathbf{R})$ via the Bargmann-Segal transform, which takes the natural basis of $\mathcal{F}$ - the orthogonal functions $z^{n}$, appropriately normalized - to the Hermite basis of $L^{2}(\mathbf{R})$. Under this transformation the operators $a^{+}$and $a^{-}$on $L^{2}(\mathbf{R})$ are respectively identified with 
the operators $M_{z}$ and $\frac{1}{r} \frac{d}{d z}$ on $\mathcal{F}$. The natural domain of $M_{z}$ is $\{f \in \mathcal{F}: z f \in \mathcal{F}\}$ and the corresponding domain of $\frac{1}{r} \frac{d}{d z}=M_{z}^{*}$ is $\left\{f \in \mathcal{F}: \bar{z} f \in L^{2}(\mathbf{C}, d \mu)\right\}$ (see e.g. [1] or [6]). See [9] for a thorough treatment of the Bargmann-Segal transform and see [3], 10], [1] for work on Toeplitz operators on $\mathcal{F}$.

Let $\mathcal{K}$ be the linear span in $\mathcal{F}$ of the reproducing kernels $e_{w}(z)=e^{r z \bar{w}}(w \in \mathbf{C})$. These functions have the property that $\left\langle f, e_{w}\right\rangle=f(w)$ for all $f \in \mathcal{F}$. Let $\mathcal{P} \mathcal{K}$ be the algebra generated by $\mathcal{K}$ together with the function $z$. Also, let $\Lambda$ be the "operator class" of Newman and Shapiro (14, [15]); this consists of those entire functions $\phi$ which satisfy $|\phi(z)|=O\left(e^{r|z|^{2} / 2-N|z|}\right)$ for all $N>0$. We will argue in the next section that the operators of multiplication by functions in $\Lambda$ are the natural solutions to $(* *)$, and their adjoints are the natural solutions to $(*)$. The following result is given in the unpublished manuscript [15]; for the reader's convenience we include the proof.

Theorem 1 ([15], §2.2). Let $\phi$ be an entire function on $\mathbf{C}$. Then $\phi \in \Lambda$ if and only if $\phi e_{w} \in \mathcal{F}$ for all $w \in \mathbf{C}$. The class $\Lambda$ is closed under differentiation.

Proof. The forward direction of the first assertion is a routine calculation. For the reverse direction, suppose $\phi e_{w} \in \mathcal{F}$ for all $w$ and fix $N>0$. Let $w_{k}=$ $(\sqrt{2} N / r) e^{2 \pi i(k / 4)}$ for $0 \leq k \leq 3$. Then

$$
\left|\phi(z) e^{r z \bar{w}_{k}}\right|=\left|\left\langle\phi e_{w_{k}}, e_{z}\right\rangle\right| \leq\left\|\phi e_{w_{k}}\right\| e^{r|z|^{2} / 2}
$$

for each $k$, by the Cauchy-Schwartz inequality. Also, for any $z \in \mathbf{C}$ there is a value of $k$ such that $-\pi / 4 \leq \operatorname{Arg}\left(z \bar{w}_{k}\right) \leq \pi / 4$, and for this $k$ we have $\operatorname{Re}\left(z \bar{w}_{k}\right) \geq$ $\left|z \bar{w}_{k}\right| / \sqrt{2}=N|z| / r$. Thus

$$
\left|\phi(z) e^{r z \bar{w}_{k}}\right|=|\phi(z)| e^{r \operatorname{Re}\left(z \bar{w}_{k}\right)} \geq|\phi(z)| e^{N|z|}
$$

and we conclude that

$$
|\phi(z)| \leq C \cdot e^{r|z|^{2} / 2-N|z|}
$$

where $C=\max _{0 \leq k \leq 3}\left\|\phi e_{w_{k}}\right\|$. This shows that $\phi \in \Lambda$.

Closure of $\Lambda$ under differentiation follows from the formula

$$
\begin{aligned}
\phi^{\prime}(w) & =\left\langle\frac{d}{d z} \phi, e_{w}\right\rangle=\left\langle\phi, r z e_{w}\right\rangle \\
& =\left(r^{2} / \pi\right) \int \bar{z} \phi(z) e^{r\left(\bar{z} w-|z|^{2}\right)} d z ;
\end{aligned}
$$

the bound $|\phi(z)|=O\left(e^{r|z|^{2} / 2-(N+1)|z|}\right)$ then yields

$$
\begin{aligned}
\left|\phi^{\prime}(w)\right| & \leq C \int|z| e^{r|z|^{2} / 2-(N+1)|z|} e^{r\left(\operatorname{Re}(z \bar{w})-|z|^{2}\right)} d z \\
& =C \int|z| e^{r|w|^{2} / 2} e^{-r|z-w|^{2} / 2} e^{-(N+1)|z|} d z \\
& =C e^{r|w|^{2} / 2-N|w|} \int|z| e^{-r|z-w|^{2} / 2-N(|z|-|w|)} e^{-|z|} d z .
\end{aligned}
$$

The final integral is finite because $|z-w| \geq|| z|-| w||$, so that the first exponent in the integrand is bounded above. Thus $\phi^{\prime}(w)=O\left(e^{r|w|^{2} / 2-N|w|}\right)$, as desired. 
We remark that $\Lambda$ is also closed under integration; this follows from an elementary estimate based on the inequality

$$
|\phi(z)| \leq|\phi(0)|+\int_{0}^{|z|}\left|\phi^{\prime}\left(t \frac{z}{|z|}\right)\right| d t
$$

For $\phi \in \Lambda$, let $M_{\phi}$ be the operator of multiplication by $\phi$, with domain $D\left(M_{\phi}\right)=$ $\{g \in \mathcal{F}: \phi g \in \mathcal{F}\}$. Thus $\mathcal{K} \subset D\left(M_{\phi}\right)$ by the preceding result, and in fact $\mathcal{P K} \subset$ $D\left(M_{\phi}\right)$ since every function in $\mathcal{P} \mathcal{K}$ is of exponential type, i.e. is $O\left(e^{N|z|}\right)$ for some $N$. The adjoint $M_{\phi}^{*}$ has similar properties:

Proposition 2. Let $\phi \in \Lambda$. Then $\mathcal{P} \mathcal{K} \subset D\left(M_{\phi}^{*}\right), \mathcal{K}$ is a core for $M_{\phi}^{*}$, and we have $M_{\phi}^{*}\left(e_{w}\right)=\bar{\phi}(w) e_{w}$ for all $w \in \mathbf{C}$.

Proof. For $w \in \mathbf{C}$ and $g \in D\left(M_{\phi}\right)$ we have

$$
\left\langle M_{\phi} g, e_{w}\right\rangle=\phi(w) g(w)=\left\langle g, \bar{\phi}(w) e_{w}\right\rangle .
$$

This shows that $\mathcal{K} \subset D\left(M_{\phi}^{*}\right)$ and $M_{\phi}^{*}\left(e_{w}\right)=\bar{\phi}(w) e_{w}$.

Let $A$ be the closure of $\left.M_{\phi}^{*}\right|_{\mathcal{K}}$. To prove that $\mathcal{K}$ is a core for $M_{\phi}^{*}$, we must show that $M_{\phi}^{*} \subset A$, or equivalently that $A^{*} \subset M_{\phi}$. Let $g \in D\left(A^{*}\right)$ and let $h=A^{*} g$. Then we have

$$
h(w)=\left\langle h, e_{w}\right\rangle=\left\langle g, A e_{w}\right\rangle=\left\langle g, \bar{\phi}(w) e_{w}\right\rangle=\phi(w) g(w)
$$

for all $w \in \mathbf{C}$. Thus $\phi g=h \in \mathcal{F}$; hence $g \in D\left(M_{\phi}\right)$, and so $A^{*} \subset M_{\phi}$ as desired.

Finally, $\mathcal{P K} \subset D\left(M_{\phi}^{*}\right)$ follows from the standard fact that $D\left(M_{\phi}^{*}\right)$ contains $\left\{f \in \mathcal{F}: \bar{\phi} f \in L^{2}(\mathbf{C}, d \mu)\right\}$. If $f \in \mathcal{P K}$, then $\bar{\phi} f$ satisfies the same growth condition as $\phi$ and hence is square-integrable, so this implies $f \in D\left(M_{\phi}^{*}\right)$.

\section{THE MAIN RESULT}

The following seems to be a reasonable notion of commutation for unbounded operators. In our setting the classes $\mathcal{K}$ and $\mathcal{P K}$ will play the role of $E$.

Definition 3. Let $E$ be a linear subspace of a Hilbert space $H$ and let $A$ and $B$ be unbounded operators on $H$ both of whose domains contain $E$, that is, $E \subset$ $D(A) \cap D(B)$. Suppose also that $E \subset D\left(A^{*}\right) \cap D\left(B^{*}\right)$. Then we say that $A$ and $B$ commute relative to $E$ and write $[A, B]=0($ rel $E$ ) if

$$
\left\langle A \eta, B^{*} \xi\right\rangle=\left\langle B \eta, A^{*} \xi\right\rangle
$$

for all $\eta, \xi \in E$.

We now present our main theorem. We regard conditions (a) and (b) as justifying the idea that operators which satisfy these conditions are the natural solutions to equation (**): (b) is the stronger version, which says that $A$ commutes with $M_{z}$ relative to the "large" domain $\mathcal{P K}$, while (a) involves the "small" domain $\mathcal{K}$ and the morally weaker condition that $A$ commutes with $M_{e_{w}}$ for all $w \in \mathbf{C}$. Likewise, conditions (f) and $(\mathrm{g})$ are weak and strong versions of equation $(*)$.

Theorem 4. Let $A$ be a closed, densely defined, unbounded operator on $\mathcal{F}$. Suppose $\mathcal{K} \subset D(A) \cap D\left(A^{*}\right)$ and $K$ is a core for $A^{*}$. Then the following are equivalent:

(a) $\left[A, M_{e_{w}}\right]=0$ (rel $\left.\mathcal{K}\right)$ for all $w \in \mathbf{C}$;

(b) $\mathcal{P K} \subset D(A) \cap D\left(A^{*}\right)$ and $\left[A, M_{z}\right]=0($ rel $\mathcal{P K})$;

(c) $A=M_{\phi}$ for some $\phi \in \Lambda$; 
(d) $A^{*} e_{w}=\bar{\phi}(w) e_{w}$ for some $\phi \in \Lambda$ and all $w \in \mathbf{C}$;

(e) $e_{w}$ is an eigenvector of $A^{*}$ for all $w \in \mathbf{C}$;

(f) $\left[\frac{d}{d z}, A^{*}\right]=0($ rel $\mathcal{K})$; and

(g) $\mathcal{P K} \subset D(A) \cap D\left(A^{*}\right)$ and $\left[\frac{d}{d z}, A^{*}\right]=0($ rel $\mathcal{P K})$.

Proof. (a) $\Rightarrow$ (c). Suppose (a) holds and let $\phi=A\left(e_{0}\right)=A(1)$. Then for any $v, w \in \mathbf{C}$ we have

$$
\left\langle A e_{v}, e_{w}\right\rangle=\left\langle M_{e_{v}} e_{0}, A^{*} e_{w}\right\rangle=\left\langle A e_{0}, M_{e_{v}}^{*} e_{w}\right\rangle=e_{v}(w)\left\langle\phi, e_{w}\right\rangle=\phi(w) e_{v}(w) ;
$$

this implies that $A e_{v}=\phi e_{v}$. Hence $\phi e_{v} \in \mathcal{F}$ for all $v$, so $\phi \in \Lambda$ follows from Theorem 1. Also $\left.A\right|_{\mathcal{K}}=\left.M_{\phi}\right|_{\mathcal{K}}$.

Let $A_{0}=\left.A\right|_{\mathcal{K}}$. Then the above shows that $A_{0} \subset M_{\phi}$ and therefore $M_{\phi}^{*} \subset A_{0}^{*}$. But also $A^{*} \subset A_{0}^{*}$, and $\mathcal{K}$ is a core for both $A^{*}$ (by hypothesis) and $M_{\phi}^{*}$ (by Proposition 2). So $A^{*}=M_{\phi}^{*}$ and hence $A=M_{\phi}$.

(c) $\Rightarrow(\mathrm{d})$. This was shown in Proposition 2 .

(d) $\Rightarrow$ (e). Vacuous.

(e) $\Rightarrow$ (a). Suppose every $e_{w}$ is an eigenvector of $A^{*}$ and fix $u, v, w \in$ C. Let $A^{*} e_{w}=\bar{\lambda} e_{w}$. Then

$$
\left\langle M_{e_{u}} e_{v}, A^{*} e_{w}\right\rangle=\lambda\left\langle e_{u+v}, e_{w}\right\rangle=\lambda e^{r(\bar{u}+\bar{v}) w}
$$

while

$$
\left\langle A e_{v}, M_{e_{u}}^{*} e_{w}\right\rangle=\left\langle A e_{v}, e^{r u \bar{w}} e_{w}\right\rangle=e^{r \bar{u} w}\left\langle e_{v}, A^{*} e_{w}\right\rangle=\lambda e^{r \bar{u} w}\left\langle e_{v}, e_{w}\right\rangle=\lambda e^{r \bar{u} w} e^{r \bar{v} w} .
$$

So $\left[A, M_{e_{u}}\right]=0($ rel $\mathcal{K})$.

(c) $\Rightarrow(\mathrm{b})$. $\mathcal{P} \mathcal{K} \subset D\left(M_{\phi}\right)$ is easy and $\mathcal{P K} \subset D\left(M_{\phi}^{*}\right)$ was shown in Proposition 2. If $\eta, \xi \in \mathcal{P K}$, then

$$
\left\langle M_{\phi} \eta, M_{z}^{*} \xi\right\rangle=\left\langle\phi \eta, M_{z}^{*} \xi\right\rangle=\langle z \phi \eta, \xi\rangle
$$

and

$$
\left\langle M_{z} \eta, M_{\phi}^{*} \xi\right\rangle=\left\langle z \eta, M_{\phi}^{*} \xi\right\rangle=\langle\phi z \eta, \xi\rangle .
$$

So $\left[M_{\phi}, M_{z}\right]=0(\operatorname{rel} \mathcal{P} \mathcal{K})$.

(b) $\Rightarrow(\mathrm{g})$. This is immediate from the fact that $M_{z}^{*}=\frac{1}{r} \frac{d}{d z}$ and the definition of commutation.

$(\mathrm{g}) \Rightarrow(\mathrm{f})$. Vacuous.

(f) $\Rightarrow(\mathrm{e})$. Suppose $\left[\frac{d}{d z}, A^{*}\right]=0($ rel $\mathcal{K})$. Then for any $v, w \in \mathbf{C}$

$$
\left\langle A^{*} e_{v}, z e_{w}\right\rangle=\left\langle\frac{1}{r} \frac{d}{d z} e_{v}, A e_{w}\right\rangle=\left\langle\bar{v} e_{v}, A e_{w}\right\rangle=\left\langle A^{*} e_{v}, v e_{w}\right\rangle .
$$

It follows that $A^{*} e_{v}$ is orthogonal to $(z-v) f$ if $f$ is any finite linear combination of the functions $e_{w}$.

In particular let

$$
f_{h}=\frac{1}{h^{n}} \sum_{k=1}^{n}(-1)^{n-k}\left(\begin{array}{l}
n \\
k
\end{array}\right) e_{k h} .
$$

Then $f_{h}$ is an $n$th order difference quotient and it converges in $\mathcal{F}$ to the $n$th Hermite polynomial $H_{n}$ as $h \rightarrow 0$. We also have $(z-v) f_{h} \rightarrow(z-v) H_{n}$ in $\mathcal{F}$. As the $H_{n}$ span all polynomials, it follows that $A e_{v}$ is orthogonal to every polynomial which vanishes at $v$. So letting $\lambda=\left\langle A e_{v}, e_{v}\right\rangle /\left\|e_{v}\right\|^{2}$, we have that $A e_{v}-\lambda e_{v}$ is orthogonal to every polynomial. Hence $A e_{v}=\lambda e_{v}$, as desired. 
Thus, if we fix the convention that $(* *)$ is the noncommutative version of the Cauchy-Riemann equations, then the unbounded operators which play the role of "noncommutative" entire functions are the operators $M_{\phi}(\phi \in \Lambda)$ - which is somewhat surprising considering that these operators all commute. Noncommutativity resides in the fact that they are not normal.

In any reasonable sense $M_{\phi}=\phi\left(M_{z}\right)$, so these are just the operators that one obtains via functional calculus by applying functions in the operator class $\Lambda$ to the creation operator. This description makes intuitive sense in our original set-up on $L^{2}(\mathbf{R})$. Thus the unbounded operators on $L^{2}(\mathbf{R})$ which satisfy (**) modulo a reasonable domain condition are precisely the operators $\phi\left(a^{+}\right)$for $\phi \in \Lambda$. However, formally defining the operator $\phi\left(a^{+}\right)$is most naturally done in the Bargmann-Segal representation. The domain $\mathcal{K}$ is interpreted in the $L^{2}(\mathbf{R})$ picture as the linear span of the eigenspaces of $a^{-}$.

The class of operators $\left\{M_{\phi}: \phi \in \Lambda\right\}$ possesses natural linear, algebraic, and topological structures isomorphic to those on $\Lambda$. In particular note that in contrast to the classical space of entire functions on the plane, $\Lambda$ is not closed under products and the class of operators which satisfy the conditions of the theorem is not closed under composition.

Remark 5. It is also possible to differentiate these operators with respect to the two noncommutative coordinates, in the sense of taking the commutator with $-\frac{1}{\hbar} P$ and $\frac{1}{\hbar} Q$. Since $Q=\left(a^{+}+a^{-}\right)$and $P=i\left(a^{+}-a^{-}\right)$, in the Bargmann-Segal representation these operators are given by $Q=\left(M_{z}+\frac{1}{r} \frac{d}{d z}\right)$ and $P=i\left(M_{z}-\frac{1}{r} \frac{d}{d z}\right)$. So if $\phi \in \Lambda$ and $\eta \in \mathcal{F}$ is of exponential type, then

$$
\begin{aligned}
{\left[\frac{1}{\hbar} Q, M_{\phi}\right] \eta } & =\frac{1}{\hbar} Q\left(M_{\phi} \eta\right)-M_{\phi}\left(\frac{1}{\hbar} Q \eta\right) \\
& =r(Q(\phi \eta)-\phi \cdot Q \eta) \\
& =r\left(z \phi \eta+\frac{1}{r}\left(\phi^{\prime} \eta+\phi \eta^{\prime}\right)-z \phi \eta-\frac{1}{r} \phi \eta^{\prime}\right) \\
& =M_{\phi^{\prime}} \eta
\end{aligned}
$$

and

$$
\begin{aligned}
{\left[-\frac{1}{\hbar} P, M_{\phi}\right] \eta } & =-\frac{1}{\hbar} P\left(M_{\phi} \eta\right)-M_{\phi}\left(-\frac{1}{\hbar} P \eta\right) \\
& =-r(P(\phi \eta)-\phi \cdot P \eta) \\
& =-i r\left(z \phi \eta-\frac{1}{r}\left(\phi^{\prime} \eta+\phi \eta^{\prime}\right)-z \phi \eta+\frac{1}{r} \phi \eta^{\prime}\right) \\
& =M_{i \phi^{\prime}} \eta .
\end{aligned}
$$

Thus differentiation of $M_{\phi}$ in the noncommutative sense corresponds to differentiation of the symbol $\phi$, and we have also verified that $M_{\phi}$ satisfies the CauchyRiemann condition in the form

$$
\left[-\frac{1}{\hbar} P, M_{\phi}\right]=i\left[\frac{1}{\hbar} Q, M_{\phi}\right]
$$

Remark 6 . We can also identify a reasonable noncommutative version of harmonic functions. Here the classical condition is $\frac{d^{2} \phi}{d z d \bar{z}}=0$, which we can formally quantize as

$$
\left[a^{+},\left[a^{-}, A\right]\right]=\left[M_{z},\left[M_{z}^{*}, A\right]\right]=0
$$


The simplest way to make this harmonicity condition precise is to invoke Theorem 4 to interpret it as asserting that $\left[M_{z}^{*}, A\right]=M_{\phi}$ for some $\phi \in \Lambda$, and then to require, minimally, that

$$
M_{z}^{*} A e_{v}-A M_{z}^{*} e_{v}=M_{\phi} e_{v}
$$

for all $v \in \mathbf{C}$. (In particular, we ask that $A e_{v} \in D\left(M_{z}^{*}\right)$ for all $v \in \mathbf{C}$; a short computation shows that this is equivalent to the requirement that $e_{v} \in D\left(\left[M_{z}^{*}, A\right]\right)$ in the sense that the map $\eta \mapsto\left\langle A e_{v}, M_{z} \eta\right\rangle-\left\langle M_{z}^{*} e_{v}, A^{*} \eta\right\rangle$ is bounded on $\mathcal{K}$.)

On $\mathcal{K}$, the solutions to $(\dagger)$ are precisely those of the form $A=M_{\Phi}+B$ where $\Phi^{\prime}=\phi$ and $B$ satisfies $\left[M_{z}^{*}, B\right]=0($ rel $\mathcal{K})$. So by Theorem $4(\mathrm{f}),\left.B\right|_{\mathcal{K}}=\left.M_{\Psi}^{*}\right|_{\mathcal{K}}$ for some $\Psi \in \Lambda$. Thus, $(\dagger)$ implies that $A=M_{\Phi}+M_{\Psi}^{*}$ on $\mathcal{K}$, for some $\Phi, \Psi \in \Lambda$.

In fact, if we assume $\mathcal{K} \subset D(A) \cap D\left(A^{*}\right)$, then $\mathcal{K}$ must be a core for $A$. For letting $A_{1}$ be the closure of $\left.\left(M_{\Phi}+M_{\Psi}^{*}\right)\right|_{\mathcal{K}}$ and $A_{2}$ the closure of $\left.\left(M_{\Phi}^{*}+M_{\Psi}\right)\right|_{\mathcal{K}}$, we have $A_{2} \subset A_{1}^{*}$ and $A_{1} \subset A_{2}^{*}$; hence $A_{1}^{*}=A_{2}$. So $A_{2} \subset A^{*}$ implies $A \subset$ $A_{1}$. We conclude that reasonable domain restrictions imply that "noncommutative harmonic operators" have $\mathcal{K}$ as a core and are of the form $M_{\Phi}+M_{\Psi}^{*}(\Phi, \Psi \in \Lambda)$ on $\mathcal{K}$. Conversely, a short computation shows that any operator of this form does satisfy $(\dagger)$. This is, of course, analogous to the fact that any function $f$, harmonic in the plane, can be written as $f=g+\bar{h}$ with $g$ and $h$ holomorphic.

If we instead begin with $\left[M_{z}^{*},\left[M_{z}, A\right]\right]=0$ as the defining condition of harmonicity, taking adjoints yields $\left[M_{z},\left[M_{z}^{*}, A^{*}\right]\right]=0$, so that (again, up to reasonable domain assumptions) $A^{*}$ is of the form $M_{\Phi}+M_{\Psi}^{*}$, and hence so is $A$.

\section{Counterexamples}

The requirement that $\mathcal{K}$ be a core for $A^{*}$ in Theorem 4 is probably unnecessary. It was conjectured in [14] (see also [5], 6]) that $\mathcal{K}$ is a core for every $M_{\phi}(\phi \in \Lambda)$, and if this is true, then our hypothesis that $\mathcal{K}$ is a core for $A^{*}$ is superfluous. For the only place that it is used is in the proof of $(\mathrm{a}) \Rightarrow(\mathrm{c})$, and if we knew that $\mathcal{K}$ was a core for $M_{\phi}$ we could reason instead as follows: we know $\left.A\right|_{\mathcal{K}}=\left.M_{\phi}\right|_{\mathcal{K}}$; hence $M_{\phi} \subset A$ since $\mathcal{K}$ is a core for $M_{\phi}$; therefore $A^{*} \subset M_{\phi}^{*}$, but $\mathcal{K} \subset D\left(A^{*}\right)$ and $\mathcal{K}$ is also a core for $M_{\phi}^{*}$; hence $A^{*}=M_{\phi}^{*}$ and so $A=M_{\phi}$.

On the other hand, the requirement $\mathcal{K} \subset D(A) \cap D\left(A^{*}\right)$ is crucial. There are operators for which this is not true but which do commute with $M_{z}$ in a reasonable sense, namely any multiplication operator $M_{\phi}$ with $\phi \notin \Lambda$.

Moving to this broader setting introduces a number of pathologies. First, observe that since $M_{z}$ is unbounded there must exist $f \in \mathcal{F}$ such that $z f \notin \mathcal{F}$. Then $1 \in$ $D\left(M_{f}\right)$ but $z \notin D\left(M_{f}\right)$. In fact, using the fact that the norm of $z^{n}$ in $\mathcal{F}$ is $r^{n / 2} \sqrt{n !}$ it is easy to check that the function $f(z)=\sum a_{n} z^{n}$ with $a_{n}=\left(r^{n} n !(n+1)^{2}\right)^{-1 / 2}$ has this property, for example. Furthermore, the function $g(z)=\sum a_{n+k} z^{n}$ satisfies $p g \in \mathcal{F}$ if $p$ is a polynomial of degree at most $k$, and $p g \notin \mathcal{F}$ if $p$ is a polynomial of degree larger than $k$.

Note that in general if $D\left(M_{\phi}\right)$ contains any polynomial $p$ of degree $k$, then it contains all polynomials of degree $\leq k$. This follows immediately from the definitions and the fact that, at $\infty,|p| \sim c|r|^{2 k}$.

In fact, given any nonnegative integer $k$ it is possible to find an entire $\phi$ so that $D\left(M_{\phi}\right)$ consists exactly of $\mathcal{P}_{k}=\{f: f$ is a polynomial of degree $\leq k\}$. A convenient way to see this is to follow the lead of Seip and Wallstén. In Proposition 2.1 of [17] they show, in our language, that if $\phi$ is the Weierstrass $\sigma$-function with period 
lattice $\left\{\sqrt{\frac{\pi}{r}}(n+i m): n, m \in \mathbf{Z}\right\}$, then $D\left(M_{\phi}\right)=\{0\}$. Suppose now that $k$ is given. Select any $k+2$ of the zeros of $\phi$ and let $p$ be a polynomial with exactly those points as its (simple) zeros. The natural modification of the argument in [17] gives

$$
D\left(M_{\phi / p}\right)=\left\{f: f \text { entire and } \int_{|z|>1}|f|^{2}|z|^{-2(k+2)} d z<\infty\right\} .
$$

This last set is $\mathcal{P}_{k}$, as required. (We note, as is pointed out in [17, that although this particular choice of $\phi$ is extremely convenient computationally, it is not essential for such an argument.)

It seems reasonable to restrict attention to the case that $M_{\phi}$ has dense domain, but there are still serious problems in this case. For any functions $f$ and $g$ of exponential type, any $w \in \mathbf{C}$ with $|w|<1$, and any $a \in\left(1-\frac{1}{2|w|}, \frac{1}{2|w|}\right)$ we have $g e^{-a w z^{2}} \in \mathcal{F}$ and

$$
\left(f e^{w z^{2}}\right)\left(g e^{-a w z^{2}}\right)=f g e^{(1-a) w z^{2}} \in \mathcal{F}
$$

since $|-a w|<\frac{1}{2}$ and $|(1-a) w|<\frac{1}{2}$. It follows that $M_{\phi}$, where $\phi=f e^{w z^{2}}$, has a "large" domain, but if $|w| \geq \frac{1}{2}$, then nothing in its domain has exponential type, and in particular $\mathcal{P} \mathcal{K}$ is disjoint from $D\left(M_{\phi}\right)$. From a comment on page 126 of [4] it appears that there are similar examples of such $\phi$ of order 2 and arbitrarily large type, such that $D\left(M_{\phi}\right)$ contains functions of order 2 and type less than $\frac{1}{2}$. Another interesting example is given in [5]; this is a function $\phi$ such that every polynomial belongs to $D\left(M_{\phi}\right)$ but $\mathcal{K} \not \subset D\left(M_{\phi}\right)$ and the polynomials are not a core of $M_{\phi}$.

For these reasons, from a purely operational point of view it clearly is desirable to require that $\mathcal{K} \subset D(A) \cap D\left(A^{*}\right)$. Some philosophical motivation may also come from the view that the coherent states $e_{w}$ play the role of the points of the noncommutative plane (see e.g. [2]).

We wish to thank Al Baernstein, James Jenkins, John McCarthy, and Harold Shapiro for helpful discussions.

\section{REFERENCES}

[1] J. Aniansson, Some Integral Representations in Real and Complex Analysis, Doctoral Thesis, Royal Institute of Technology, Stockholm (1999).

[2] S. T. Ali, J.-P. Antoine, J.-P. Gazeau, and U. A. Mueller, Coherent states and their generalizations: a mathematical overview, Rev. Math. Phys. 7 (1995), 1013-1104. MR 97b:81040

[3] C. A. Berger and L. A. Coburn, Toeplitz operators on the Segal-Bargmann space, Trans. Amer. Math. Soc. 301 (1987), 813-829. MR 88c:47044

[4] R. Boas, Entire Functions, Academic Press (1954). MR 16:914f

[5] A. A. Borichev, The polynomial approximation property in Fock-type spaces, Math. Scand. 82 (1998), 256-264. CMP 99:01

[6] D. Cichoń and J. Stochel, On Toeplitz operators in Segal-Bargmann spaces, Univ. Iagel. Acta Math. 34 (1997), 35-43. MR 98d:47061

[7] A. Connes, Noncommutative geometry, Academic Press (1995). MR 95j:46063

[8] E. G. Effros, Advances in quantized functional analysis, Proc. ICM (Berkeley, California, 1986), 906-916, AMS (1987). MR 89e:46064.

[9] G. B. Folland, Harmonic Analysis in Phase Space, Princeton (1989). MR 92k:22017

[10] J. Janas, Unbounded Toeplitz operators in the Bargmann-Segal space, Studia Math. 99 (1991), 87-99. MR 92j:47046

[11] J. Janas and J. Stochel, Unbounded Toeplitz operators in the Segal-Bargmann space, II, J. Funct. Anal. 126 (1994), 418-447. MR 95m:47040

[12] J. E. Moyal, Quantum mechanics as a statistical theory, Proc. Cambridge Philos. Soc. 45 (1949), 99-124. MR 10:582d 
[13] J. von Neumann, Die eindeutigkeit der Schrödingerschen operatoren, Math. Ann. 104 (1931), $570-578$.

[14] D. J. Newman and H. S. Shapiro, Fischer spaces of entire functions, in Entire Functions and Related Parts of Analysis (J. Koorevaar, ed.), AMS Proc. Symp. Pure Math. XI (1968), 360-369. MR 38:2333

[15] A Hilbert space of entire functions related to the operational calculus, manuscript.

[16] M. A. Rieffel, Non-commutative tori - a case study of non-commutative differentiable manifolds, Contemp. Math. 105 (1990), 191-211. MR 91d:58012

[17] K. Seip and R. Wallstén, Density theorems for sampling and interpolation in the BargmannFock space. II, J. Reine Angew. Math. 429 (1992), 107-113. MR 93g:46026b

[18] S. L. Woronowicz, Differential calculus on compact matrix pseudogroups (quantum groups), Comm. Math. Phys. 122 (1989), 125-170. MR 90g:58010

Department of Mathematics, Washington University, St. Louis, Missouri 63130

E-mail address: rr@math.wustl.edu

Department of Mathematics, Washington University, St. Louis, Missouri 63130

E-mail address: nweaver@math.wustl.edu 